\title{
Public awareness of the neurological manifestation of COVID-19 in Saudi Arabia
}

Amal M. Alkhotani, MBBCH, Tala S. AlSindi, Medical Student, Atheer A. Alqurashi, Medical Student, Roaa M. Masarit, Medical Student, Ruba T. Gazzaz, Medical Student, Renad Z. Saggat, Medical Student, Mayar A. Halawani, Medical Student.

\begin{abstract}
الأهداف: دراسة وعي الشعب السعودي بالأعراض العصبية المصاحبة لعدوي دماف : COVIID-19.

المنهجية : كانت هذه دراسة مقطعية تم إجراؤها باستخدام استطلاع السكان Google Form

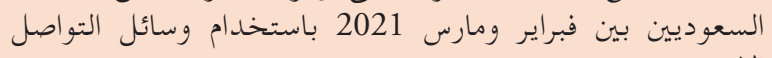

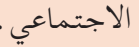

النتائج : أكمل ما مجموعه 831 مشار كا الاستبيان . تم تقييم توزيع العمديع

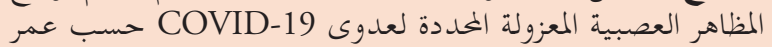

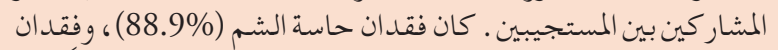

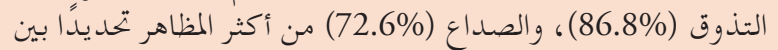

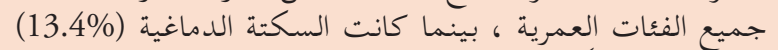

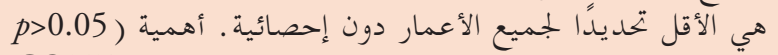

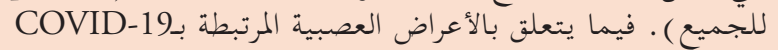

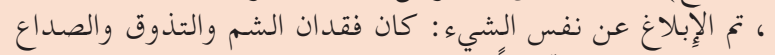

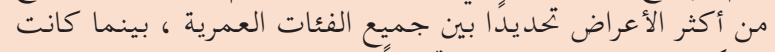

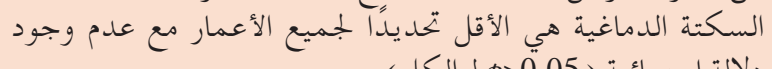
دلالة إحصائية (

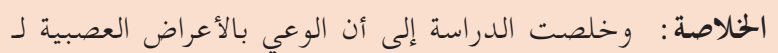

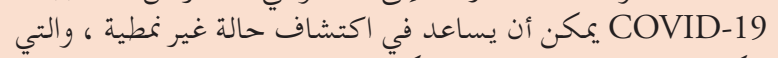

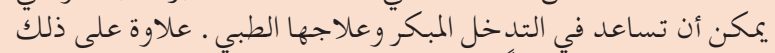

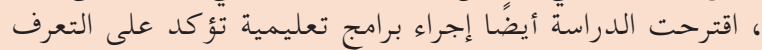

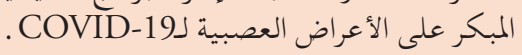

Objectives: To assess awareness of the neurological manifestation of COVID-19 on the Saudi population.

Methods: This was a cross-sectional study conducted using a Google Form survey to obtain responses randomly from the Saudi population between February and March 2021 using social media.

Results: A total of 831 participants completed the questionnaire. The distribution of the identified isolated neurological manifestations of COVOD-19 infections by participants' age was assessed among the respondents. Loss of smell $(88.9 \%)$, loss of taste $(86.8 \%)$, and headache $(72.6 \%)$ were the most identified first manifestations among all the age groups, while stroke $(13.4 \%)$ was the least identified for all ages with no statistical significance ( $p>0.05$ for all). Regarding COVID-19 related neurological symptoms, the same was reported: loss of smell, taste, and headache were the most identified symptoms among all the age groups, while stroke was the least identified for all ages with no statistical significance $(p>0.05$ for all).

Conclusion: The study concluded that awareness of COVID-19's neurological symptoms could help detect an atypical case, which can help in early intervention and its medical treatment. Moreover, the study also suggested conducting educational programs that emphasize the early identification of neurological symptoms of COVID-19.

Neurosciences 2022; Vol. 27 (1): 10-15 doi: $10.17712 / n s j .2022 .1 .20210089$

From the Department of Medicine (Alkhotani), Umm AlQura University, from Faculty of Medicine (AlSindi, Alqurashi, Masarit, Gazzaz, Saggat, Halawani), Umm AlQura University, Makkah, Kingdom of Saudi Arabia.

Received 14th July 2021. Accepted 20th September 2019.

Address correspondence and reprint request to: Dr. Amal M Alkhotani, Department of Medicine, Umm AlQura University, Makkah, Kingdom of Saudi Arabia.E-mail: Dr.amalalkhotani@hotmail.com, ORCID ID: https://orcid.org/0000-0001-8310-1875

COVID-19's first case was identified in 2019 in Wuhan city of China, and then in the year 2020, this spread globally, causing a pandemic. ${ }^{1,2}$ It is found that the COVID-19 virus does not find its way into the respiratory system as other respiratory viruses do. This virus is transmitted through various means such as via droplets, airborne, and even through surfaces, 
and at any time; therefore, it is very difficult to control the virus from spreading. ${ }^{2,3}$ This virus can affect various bodily functions, causing multiple symptoms, and eventually lead to acute organ damage or even endless sequels. Although the most common manifestation is fever and respiratory tract symptoms, extrapulmonary symptoms include hematological problems, cardiac arrest, acute kidney injury, gastrointestinal pain, liver damage, neurological illnesses, ocular manifestation, and dermatologic complications. ${ }^{2,4,5}$ Several studies have shown that patients of COVID-19 could show signs of the central or peripheral nervous system that can vary from mild to severe (loss of taste and smell, headache, dizziness, ischemic stroke, delirium, and encephalitis). These symptoms can appear before the start of fever, respiratory tract, or as an isolated manifestation. ${ }^{6-9}$ Therefore, this study has evaluated public awareness about the neurological appearances of COVID-19 among the Saudi population.

Methods. Study design and timeframe. This study was cross-sectional and conducted using an electronic survey to get responses from the Saudi population randomly. This study was conducted between FebruaryMarch 2021.

Study population. The sample study included Saudi people aged above 15 years whether they had COVID-19 infection or not. The study included 831 participants who completed the online survey questionnaire. The study excluded participants who were already diagnosed with neurological disorders and incomplete responses.

Sampling methodology. The sample size was determined via Raosoft online calculator. The recommended sample size was 385 with a confidence interval of $95 \%$ and a $5 \%$ margin of error. The questionnaire was self-administered and comprised of 4 sections. The first section of the study included the demographic details of the participants, the second section comprised of questions about previous exposure to COVID-19, the third section consisted of the medical history of the respondents, and the last section focused upon awareness, knowledge, information sources, and opinion about the appearance of neurological symptoms of COVID-19. The survey was accessible via a link, and additional information material was provided for further clarification and understanding

Disclosure. Authors have no conflict of interests, and the work was not supported or funded by any drug company. of the survey to the study participants. Also, to assess the readability of the questionnaire, a faculty member was selected to serve the purpose. Moreover, a pilot study was also carried out consisting of 8 middle-aged randomly selected participants to check the reliability of the questionnaire. Their responses were taken into consideration, and those minor changes were made to the final questionnaire. Finally, the questionnaire was also reviewed and approved by 2 certified neurologists. ${ }^{10}$

Ethical considerations. An ethical approval of this study was taken from the Biomedical Research Ethics Committee at Umm Al Qura University, Makkah, Saudi Arabia (No: NGMX150221). A written consent form was obtained from all the study participants after a thorough explanation of the aim of the study.

Data analysis. After collecting data, it was modified, coded, and entered into Statistical Package of Social Sciences version 22.0 (SPSS, Inc, Chicago, IL). A two-tailed test was carried out for statistical analysis of data. A $p$-value less than 0.05 was considered to be statistically significant. ${ }^{11}$ Also, descriptive statistical analysis based on percentage and frequency was also

Table 1 - Demographic details of the participants.

\begin{tabular}{lcc}
\hline Demographic details & No. & $(\%)$ \\
\hline Age in years & & \\
$18-25$ & 369 & $(44.4)$ \\
$26-34$ & 120 & $(14.4)$ \\
$35-44$ & 144 & $(17.3)$ \\
$45-54$ & 143 & $(17.2)$ \\
$55+$ & 55 & $(6.6)$ \\
Gender & & \\
Male & 151 & $(18.2)$ \\
Female & 680 & $(81.8)$ \\
Education level & & \\
Below high school & 26 & $(3.1)$ \\
High school & 240 & $(28.9)$ \\
University or above & 565 & $(68.0)$ \\
Do you suffer from any chronic disease? & & \\
Yes & 38 & $(4.6)$ \\
No & 793 & $(95.4)$ \\
Have you ever been diagnosed with neurological diseases? \\
Yes \\
No \\
Ifyes, mention \\
Diabetic neuropathy \\
Migraine headache \\
Multiple sclerosis \\
Sciatica \\
Trigeminal neuralgia & 825 & $(.7)$ \\
$\quad$ & & \\
\hline & 1 & $(.1)$ \\
& 1 & $(.1)$ \\
& 1 & $(.1)$ \\
& 1 & $(.1)$ \\
& 1 & $(.1)$ \\
\hline
\end{tabular}




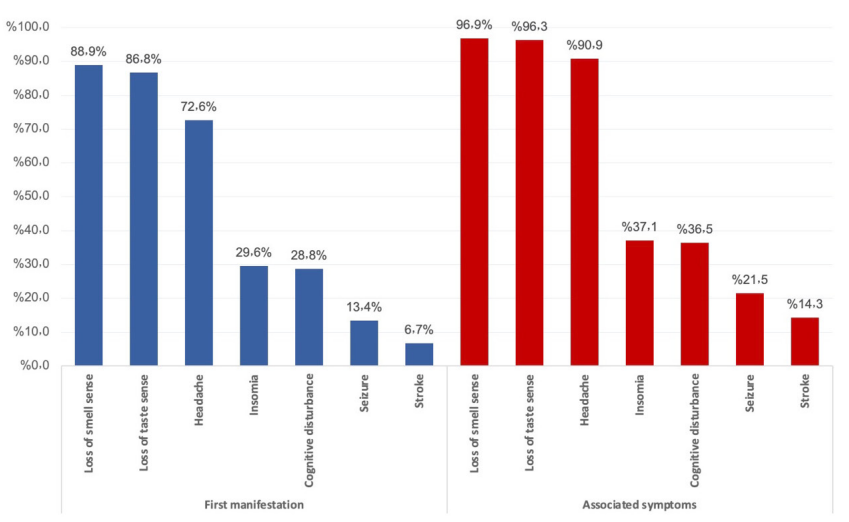

Figure 1 - Public awareness of neurological symptom's associated with COVID-19 in Saudi Arabia.

Table 2 - COVID-19 infection history and relevant data among the participants.

\begin{tabular}{lcc}
\hline COVID-19 infection history & No. & $(\%)$ \\
\hline Have you ever been infected with coronavirus & COVID-19)? \\
Yes & 148 & $(17.8)$ \\
No & 683 & $(82.2)$ \\
What was the initial symptom that you suffered from? \\
No symptoms & 6 & $(4.1)$ \\
Fever & 42 & $(28.4)$ \\
Lethargy and fatigue & 23 & $(15.5)$ \\
Headache & 19 & $(12.8)$ \\
Sore throat & 13 & $(8.8)$ \\
Cough & 9 & $(6.1)$ \\
Runny nose & 8 & $(5.4)$ \\
Anosmia & 7 & $(4.7)$ \\
Shivering and pain in the body & 7 & $(4.7)$ \\
Diarrhea & 4 & $(2.7)$ \\
Shortness of breath & 4 & $(2.7)$ \\
Loss of taste & 3 & $(2.0)$ \\
Dizziness & 2 & $(1.4)$ \\
Back pain and general body pain & 1 & $(.7)$ \\
Do you know anyone who has previously been infected with coronavirus \\
(COVID-19)?
\end{tabular}

carried out for all the study variables. Furthermore, cross-tabulation was carried out to investigate the distribution of the recognized first manifestation and other COVID-19 symptoms by the respondent's age, gender, and educational level. A Pearson correlation and Chi-square test were carried out to investigate the significance of relationships.
Results. The study included 831 participants who completed the survey questionnaire. The age of the respondents ranged from 18-65 years with a mean value of $34.8 \pm 12.4$ years. Altogether, $680(81.8 \%)$ participants were women, and 565 (68\%) were university graduates or above. Among study participants, 38 (4.6\%) had chronic health problems (asthma, DM, HTN, and others). In contrast, only 5 (0.6\%) had been diagnosed with neurological diseases: diabetic neuropathy (1 case), migraine (1 case), multiple sclerosis (1 case), and trigeminal neuralgia (1 case) (Table 1$)$.

Table 2 shows the COVID-19 infection history and relevant data of study participants. The most-reported initial symptoms were fever (28.4\%), followed by lethargy and fatigue $(15.5 \%)$, headache $(12.8 \%)$, sore throat $(8.8 \%)$, cough $(6.1 \%)$ runny nose $(5.4 \%)$, and anosmia $(4.7 \%)$. At the same time, back pain and general body pain were the least reported (1 case).

Figure 1 illustrates the public awareness of COVID19-associated with neurological manifestations in the Saudi Arabia population. As for the isolated neurological manifestations, loss of smell was the most reported $(88.9 \%)$, followed by loss of taste $(86.8 \%)$, headache $(72.6 \%)$, insomnia $(29.6 \%)$, cognitive disturbance $(28.8 \%)$, seizures $(13.4 \%)$, and stroke $(6.7 \%)$. The neurological symptoms such as loss of smell were the most reported $(96.9 \%)$, followed by loss of taste $(96.3 \%)$, headache $(90.9 \%)$, insomnia $(37.1 \%)$, cognitive disturbance $(36.5 \%)$, seizures $(21.5 \%)$, and stroke (14.3\%).

Table 3 shows the distribution of the identified initial neurological symptoms manifestation of COVID-19 by participants' age. Loss of smell, taste, and headache 
Table 3 - Distribution of the identified first neurological manifestations and symptoms associated with COVID-19 by participants' age.

\begin{tabular}{|c|c|c|c|c|c|c|c|c|c|c|c|}
\hline \multirow{3}{*}{ Domain/Item } & \multicolumn{10}{|c|}{ Age (years) } & \multirow[t]{3}{*}{$P$-value } \\
\hline & \multicolumn{2}{|c|}{$18-25$} & \multicolumn{2}{|c|}{$26-34$} & \multicolumn{2}{|c|}{$35-44$} & \multicolumn{2}{|c|}{$45-54$} & \multicolumn{2}{|c|}{$55+$} & \\
\hline & No. & $(\%)$ & No. & $(\%)$ & No. & $(\%)$ & No. & (\%) & No. & $(\%)$ & \\
\hline \multicolumn{12}{|l|}{ First manifestation } \\
\hline Headache & 281 & $(76.2)$ & 86 & $(71.7)$ & 98 & $(68.1)$ & 98 & $(68.5)$ & 40 & $(72.7)$ & .280 \\
\hline Loss of smell & 335 & $(90.8)$ & 107 & $(89.2)$ & 124 & $(86.1)$ & 124 & $(86.7)$ & 49 & $(89.1)$ & .529 \\
\hline Loss of Taste & 327 & $(88.6)$ & 103 & $(85.8)$ & 122 & $(84.7)$ & 122 & $(85.3)$ & 47 & $(85.5)$ & .725 \\
\hline Cognitive disturbance & 114 & $(30.9)$ & 33 & $(27.5)$ & 41 & $(28.5)$ & 38 & $(26.6)$ & 13 & $(23.6)$ & .744 \\
\hline Seizure & 51 & $(13.8)$ & 15 & $(12.5)$ & 20 & $(13.9)$ & 18 & $(12.6)$ & 7 & $(12.7)$ & .992 \\
\hline Stroke & 24 & $(6.5)$ & 11 & $(9.2)$ & 10 & (6.9) & 9 & $(6.3)$ & 2 & (3.6) & .726 \\
\hline Insomnia & 106 & $(28.7)$ & 40 & $(33.3)$ & 34 & $(23.6)$ & 50 & $(35.0)$ & 16 & $(29.1)$ & .249 \\
\hline \multicolumn{12}{|l|}{ Symptom Associated } \\
\hline Headache & 328 & $(88.9)$ & 113 & $(94.2)$ & 132 & $(91.7)$ & 129 & $(90.2)$ & 53 & $(96.4)$ & .240 \\
\hline Loss of smell & 351 & $(95.1)$ & 115 & $(95.8)$ & 142 & $(98.6)$ & 138 & $(96.5)$ & 54 & $(98.2)$ & .380 \\
\hline Loss of Taste & 356 & $(96.5)$ & 115 & $(95.8)$ & 144 & $(100.0)$ & 136 & $(95.1)$ & 54 & $(98.2)$ & .133 \\
\hline Cognitive disturbance & 143 & $(38.8)$ & 39 & $(32.5)$ & 52 & $(36.1)$ & 56 & $(39.2)$ & 13 & $(23.6)$ & .198 \\
\hline Seizure & 91 & $(24.7)$ & 18 & $(15.0)$ & 30 & $(20.8)$ & 31 & $(21.7)$ & 9 & $(16.4)$ & .193 \\
\hline Stroke & 65 & $(17.6)$ & 17 & $(14.2)$ & 16 & $(11.1)$ & 18 & $(12.6)$ & 3 & $(5.5)$ & .080 \\
\hline Insomnia & 146 & $(39.6)$ & 40 & $(33.3)$ & 49 & $(34.0)$ & 55 & $(38.5)$ & 18 & $(32.7)$ & .585 \\
\hline \multicolumn{12}{|c|}{$P$ - Pearson $X^{2}$ test } \\
\hline
\end{tabular}

were the most identified first manifestations among all the age groups, while stroke was the least identified for all ages with no statistical significance $(p>0.05$ for all). Regarding neurological symptoms of COVID-19, the same was reported: loss of smell, loss of taste, and headache were the most identified symptoms among all the age groups, while stroke was the least identified for all ages with no statistical significance $(p>0.05$ for all).

Table 4 presents the distribution of the identified first neurological manifestations of COVID-19 by participants' gender. As for age, loss of smell, taste, and headache were the most identified first manifestations among male and female participants, with higher rates among women than men and statistical significance for headache $(74.7 \%$ vs. $62.9 \%$, respectively; $p=0.003)$. For the neurological symptoms associated, the same results were reported.

Table 5 shows the distribution of the identified isolated neurological symptoms and manifestations of COVID-19 by participants' education level. Like the previous findings, loss of taste, smell, and headache were the most identified first manifestations among all education levels but with higher rates among those with education below high school with statistical significance for all manifestations except for headache, stroke, and insomnia. Seizures were reported by $26.9 \%$ of the low educated category, significantly higher than that of the highly educated group $(18.8 \% ; p=0.018)$. In addition, $46.2 \%$ of the low-educated group reported insomnia
Table 4 - Distribution of the identified first neurological manifestations and symptoms associated with COVID-19 by participants' sex.

\begin{tabular}{lccccc}
\hline \multicolumn{5}{c}{ Male } & \multicolumn{2}{c}{ Female } & $P$-value \\
& $\mathrm{N}$ & $(\%)$ & $\mathrm{N}$ & $(\%)$ & \\
\hline First manifestation & & & & & \\
Headache & 95 & $(62.9)$ & 508 & $(74.7)$ & $.003^{*}$ \\
Loss of smell & 129 & $(85.4)$ & 610 & $(89.7)$ & .130 \\
Loss of Taste & 124 & $(82.1)$ & 597 & $(87.8)$ & .063 \\
Cognitive disturbance & 40 & $(26.5)$ & 199 & $(29.3)$ & .496 \\
Seizure & 27 & $(17.9)$ & 84 & $(12.4)$ & .071 \\
Stroke & 12 & $(7.9)$ & 44 & $(6.5)$ & .513 \\
Insomnia & 50 & $(33.1)$ & 196 & $(28.8)$ & .296 \\
$\quad$ Symptom Associated & & & & & \\
Headache & 133 & $(88.1)$ & 622 & $(91.5)$ & .191 \\
Loss of smell & 136 & $(90.1)$ & 664 & $(97.6)$ & $.001^{*}$ \\
Loss of Taste & 143 & $(94.7)$ & 662 & $(97.4)$ & .091 \\
Cognitive disturbance & 60 & $(39.7)$ & 243 & $(35.7)$ & .356 \\
Seizure & 39 & $(25.8)$ & 140 & $(20.6)$ & .157 \\
Stroke & 22 & $(14.6)$ & 97 & $(14.3)$ & .923 \\
Insomnia & 66 & $(43.7)$ & 242 & $(35.6)$ & .062 \\
\hline \multicolumn{5}{l}{$\quad$ P: Pearson $\mathrm{X}^{2}$ test, ${ }^{*} p<0.05$ (significant) } \\
\hline
\end{tabular}

compared with $33.6 \%$ of the highly educated group $(p=0.11)$.

Discussion. This community-based study investigated public awareness about the occurrence of 
Table 5 - Distribution of the identified first neurological manifestations and symptoms associated with COVID-19 by participants' education level.

\begin{tabular}{|c|c|c|c|c|c|c|c|}
\hline \multirow{3}{*}{ Domain / Item } & \multicolumn{6}{|c|}{ Education level } & \multirow{3}{*}{$P$-value } \\
\hline & \multicolumn{2}{|c|}{ Below high school } & \multicolumn{2}{|c|}{ High school } & \multicolumn{2}{|c|}{ University or above } & \\
\hline & No. & $(\%)$ & No. & $(\%)$ & No. & $(\%)$ & \\
\hline \multicolumn{8}{|l|}{ First manifestation } \\
\hline Headache & 23 & $(88.5)$ & 178 & $(74.2)$ & 402 & $(71.2)$ & .124 \\
\hline Loss of smell & 25 & $(96.2)$ & 224 & $(93.3)$ & 490 & $(86.7)$ & $.012^{*}$ \\
\hline Loss of Taste & 23 & $(88.5)$ & 221 & $(92.1)$ & 477 & $(84.4)$ & $.013^{*}$ \\
\hline Cognitive disturbance & 12 & $(46.2)$ & 78 & $(32.5)$ & 149 & $(26.4)$ & $.029^{*}$ \\
\hline Seizure & 6 & $(23.1)$ & 42 & $(17.5)$ & 63 & $(11.2)$ & $.018^{*}$ \\
\hline Stroke & 2 & $(7.7)$ & 18 & $(7.5)$ & 36 & $(6.4)$ & .827 \\
\hline Insomnia & 12 & $(46.2)$ & 79 & $(32.9)$ & 155 & $(27.4)$ & .054 \\
\hline \multicolumn{8}{|l|}{ symptom Associated } \\
\hline Headache & 25 & $(96.2)$ & 214 & $(89.2)$ & 516 & (91.3) & .369 \\
\hline Loss of smell & 25 & $(96.2)$ & 226 & $(94.2)$ & 549 & $(97.2)$ & .121 \\
\hline Loss of Taste & 24 & $(92.3)$ & 232 & $(96.7)$ & 549 & $(97.2)$ & .371 \\
\hline Cognitive disturbance & 10 & $(38.5)$ & 96 & $(40.0)$ & 197 & (34.9) & .375 \\
\hline Seizure & 7 & (26.9) & 66 & $(27.5)$ & 106 & (18.8) & $.018^{*}$ \\
\hline Stroke & 5 & $(19.2)$ & 41 & (17.1) & 73 & (12.9) & .234 \\
\hline Insomnia & 12 & $(46.2)$ & 106 & $(44.2)$ & 190 & $(33.6)$ & $.011^{*}$ \\
\hline
\end{tabular}

neurological symptoms associated with COVID-19. Though the most common symptoms of COVID-19 are not neurological, several studies have focused on the appearance of neurological symptoms of the disease. This manifestation of the neurological symptoms can be divided into mild and severe, where the affected can experience anosmia, loss of taste, headache, dizziness, ischemic stroke, and encephalitis. ${ }^{6}$ Studies have reported the appearance of some other neurological symptoms such as anorexia $(37.7 \%)$, myalgia $(33.0 \%)$, and diarrhea $(32.7 \%) .{ }^{11}$ The current study has divided neurological symptoms into CNS problems, muscular injuries, and PNS problems. Patients with a severeillness suffered CNS and muscle damage symptoms. ${ }^{12}$ In more than $35 \%$ of cases, the patients experienced neurological symptoms. Many COVID-19 patients reported experiencing the appearance of neurological symptoms as the first manifestation of the virus. However, COVID-19 patients highly infected with the virus showed a high prevalence of neurological symptoms, which could cause cerebral hypoxia, which occurs due to respiratory failure. ${ }^{13}$ One of the studies detected a new on-set of neurological symptoms in 106 patients out of 391 $(27.1 \%)$ irrespective of the on-set of other symptoms. The most commonly reported neurological symptoms among these individuals changed in taste (33.1\%), change in smell $(24.5 \%)$, and headache $(22.6 \%)$, and these conditions were experienced as initial ones among 38 (9.7\%) patients. ${ }^{14}$ Among the many neurological symptoms reported by the patients, loss of smell was the most commonly experienced one (996.9\%), which was followed by loss of taste $(96.3 \%)$, headache $(90.9 \%)$, insomnia (37.1\%), cognitive disturbance (36.5\%), seizures $(21.5 \%)$, and stroke $(14.3 \%)$. The study found that among all the neurological symptoms, loss of taste, smell, and headache occurred most commonly among all age levels. In contrast, stroke was the least common symptom for all age levels, with no statistical significance $(p>0.05)$. Another study reported that $2.5 \%$ of the patients experienced cerebrovascular disease (ischemic stroke, cerebral venous thrombosis, and intracranial hemorrhage). These patients, upon being tested, should have high levels of CK and LDH, which are markers of skeletal muscle injury, especially in the severe subcategory. One-third of the study patients experienced the severity of the disease, and one-fifth of them were hospitalized and admitted to the intensive care unit (ICU). ${ }^{15}$

At the education level, the study found that loss of smell, taste, and headache were the most identified symptoms among the different educational levels, while stroke was the least identified for all with a high rate among the highly educated group for the 3 symptoms. This was attributed to less educated participants as 
they were more afraid of the disease. They followed the news, asked other people, and this made them more knowledgeable. Secondly, less educated people are more exposed to information coming from other contacts and side talking which may have helped them be aware of the virus. The study, therefore, found that awareness about the neurological symptoms of COVID-19 can lead to early diagnosis, which can help in intervention and medical treatment to assist in reducing disease implications and prognosis. The pathophysiology of the association between COVID-19 and neurological symptoms is still under investigation and needs attention. The general population and physicians should know this possible association to prevent acute respiratory syndrome coronavirus two infections. ${ }^{12}$ For that, public awareness will play a vital role, especially about the occurrence of stroke, since knowledge is lacking. A health education program is needed to establish a satisfactory level of knowledge.

Study limitations. The study was subjected to several limitations. Firstly, the sample size was small as compared to other studies. Secondly, the study used an electronic questionnaire that may lead to a recall bias. There was also the absence of an interviewer, which may have led to participants misunderstanding certain points.

The reduced sample size was due to the electronic survey. Also, the study experienced more responses from the female population compared to the male population.

Conclusion. This study evaluated knowledge about the occurrence of neurological symptoms related to COVID-19 among the Saudi population. According to our data, there was adequate knowledge about the loss of smell $(88.9 \%)$, loss of taste $(86.8 \%)$, headache $(72.6 \%)$. However, there is inadequate knowledge about the association with insomnia (29.6\%), seizures (13.4\%), cognitive disturbance $(28.8 \%)$, and stroke $(6.7 \%)$. There is a need for greater public awareness about the incidence of stroke. Educational programs must be established to emphasize the importance of the early recognition of COVID-19 symptoms (neurological and non-neurological). These may be carried out through social media, TV advertising, national awareness programs, and campaigns. Though the study is population-based, the sample size was not representative of the selected design. Also, the female population participated more compared to male participants. Further study has a large sample size recommended to evaluate the awareness of neurological symptoms of COVID-19.

\section{References}

1. Whittaker A, Anson M, Harky A. Neurological Manifestations of COVID-19: A systematic review and current update. Acta Neurol Scand 2020; 142: 14-22.

2. Wang F, Kream RM, Stefano GB. Long-Term Respiratory and Neurological Sequelae of COVID-19. Med Sci Monit 2020; 26: e928996.

3. Oldfield E, Malwal SR. COVID-19 and Other Pandemics: How Might They Be Prevented? ACS Infect Dis 2020; 6: 15631566.

4. Revzin MV, Raza S, Srivastava NC, Warshawsky R, D’Agostino C, Malhotra A, et al. Multisystem Imaging Manifestations of COVID-19, Part 2: From Cardiac Complications to Pediatric Manifestations. Radiographics 2020; 40: 1866-1892.

5. Lai C-C, Ko W-C, Lee P-I, Jean S-S, Hsueh P-R. Extrarespiratory manifestations of COVID-19. Int J Antimicrob Agents 2020; 56: 106024.

6. Wenting A, Gruters A, van Os Y, Verstraeten S, Valentijn S, Ponds R, et al. COVID-19 Neurological Manifestations and Underlying Mechanisms: A Scoping Review. Front Psychiatry 2020; 11: 860 .

7. Beach SR, Praschan NC, Hogan C, Dotson S, Merideth F, Kontos N, et al. Delirium in COVID-19: A case series and exploration of potential mechanisms for central nervous system involvement. Gen Hosp Psychiatry 2020; 65: 47-53.

8. Kopscik MR, Giourgas BK, Presley BC. A Case Report of Acute Motor and Sensory Polyneuropathy as the Presenting Symptom of SARS-CoV-2. Clin Pract Cases Emerg Med 2020; 4: 352-354.

9. Pinzon RT, Wijaya VO, Buana RB, Al Jody A, Nunsio PN. Neurologic Characteristics in Coronavirus Disease 2019 (COVID-19): A Systematic Review and Meta-Analysis. Front Neurol 2020; 11: 565.

10. Alluqmani MM, Almshhen NR, Alotaibi RA, Aljardi OY, Zahid HM. Public Awareness of Ischemic Stroke in Medina city, Kingdom of Saudi Arabia. Neurosciences (Riyadh) 2021; 26: $134-140$.

11. Gupta I, Reddy MK, Hussain MM, Murthy PM, Robert CA. Atypical Neurological Manifestations of COVID-19. Cureus 2020; 12: e8518-e8518.

12. Di Carlo DT, Montemurro N, Petrella G, Siciliano G, Ceravolo R, Perrini P. Exploring the clinical association between neurological symptoms and COVID-19 pandemic outbreak: a systematic review of current literature. J Neurol 2021; 268: 1561-1569.

13. Niazkar HR, Zibaee B, Nasimi A, Bahri N. The neurological manifestations of COVID-19: a review article. Neurol Sci 2020; 41: 1667-1671.

14. Garg R, Jain R, Sodani A, Chouksey D, Dosi R, Athale S, et al. Neurological Symptoms as Initial Manifestation of Covid-19 - An Observational Study. Ann Indian Acad Neurol 2020; 23 : 482-486.

15. Yassin A, Nawaiseh M, Shaban A, Alsherbini K, El-Salem K, Soudah O, et al. Neurological manifestations and complications of coronavirus disease 2019 (COVID-19): a systematic review and meta-analysis. BMC Neurol 2021; 21: 138. 